\title{
Riesgos de la radiación en imágenes pediátricas.
}

\section{Radiation risks in pediatric images}

\author{
Dra Susana Renedo de la Hoz \\ Radiólogo Pediatra \\ Hospital Clínico San Borja - Arriarán
}

\begin{abstract}
RADIATION RISK IN PEDIATRIC IMAGES
The advance of medical imaging technology has led to an increase in the medical radiation exposure, especially derived from computed tomography (CT).

Recent studies confirm a small but significant increase of cancer cases induced by CT radiation. Children are markedly more sensitive to radiation than adults and in addition, their life expectancy is longer, so we must use all resources to optimize and reduce the exposure dose using the ALARA concept. CT is an important diagnostic tool in medical practice and its benefits far outweigh the costs of radiation if the indication is properly justified.
\end{abstract}

Key words: radiobiology, cancer radiation induced, risk assessment

\section{RESUMEN}

El avance tecnológico de las imágenes para evaluación de enfermedades ha llevado a un aumento considerable de la radiación de origen médica, principalmente la proveniente de la tomografía computada (TC)

Estudios recientes confirman un pequeño pero significativo incremento de casos de cáncer inducidos por radiación generada por la TC. Siendo los niños reconocidamente más sensibles a la radiación que los adultos y sumado a su mayor expectativa de vida, es que debemos usar todos los recursos para optimizar y reducir la dosis de exposición aplicando el concepto de ALARA. La TC es una herramienta diagnóstica importantísima en la práctica médica, y sus beneficios superan ampliamente los costos de la radiación si su indicación está adecuadamente justificada.

Palabras clave: radiobiología, efectos de la radiación, medición de riesgo

\section{INTRODUCCIÓN}

Los estudios de imágenes han avanzado notablemente desde los inicios de la radiología, cuando Roentgen y su esposa descubrieron una luz incandescente que lograba atravesar el cuerpo humano, y la llamaron Rayos X (por su naturaleza desconocida). Luego comenzó la preocupación por los efectos adversos que éstos podrían producir. La Sra. Roentgen, que muy románticamente pasaba noches con su marido admirando la luz de su nuevo descubrimiento, años más tarde moriría por causa de una leucemia probablemente inducida por la radiación(1).

Los avances tecnológicos han hecho que los equipos de rayos convencionales sean cada vez más eficientes, pudiendo regular y disminuir la radiación entregada al paciente y al equipo médico, aportando también protección y aislamiento de los lugares con fuentes de radiación $(2,3)$.

El gran salto de la imagenología se produjo a inicios de años 70 cuando Hounsfield desarrolló la Tomografía Computada (TC) logrando visualizar órganos que antes la radiología convencional no lograba hacerlo, como el cerebro. Rápidamente se masificó su uso y progresó a la tomografía multicorte, más rápido y con imágenes multiplanares. De la mano del desarrollo tecnológico creció aún más la preocupación por los efectos de la radiación (4).

El año 2001 el diario USA TODAY publicó un artículo que causó gran alarma pública. Estudios científicos indicaban que la TC podía causar cáncer en los niños y además que éstos recibían dosis mucho más alta de la necesaria. La Sociedad

\section{Correspondencia:}

Dra Susana Renedo de la Hoz

Hospital Clínico San Borja - Arriarán

Santa Rosa1234 - Santiago

E-mail: susana.renedo@gmail.com
Americana de Radiología Pediátrica, citó una conferencia multidisciplinaria, donde se propuso el concepto de ALARA (As Low As Reasonable Achievable) para el uso razonable de la TC (2).

El año 2008 se inició una campaña educativa enfocada a tomar conciencia del aumento de los niveles de radiación por TC, la que se conoce al día de hoy como Image Gently $(2,3)$.

\section{RAYOS X, UNIDADES Y DOSIS DE RADIACIÓN}

Los rayos $X$ son un tipo de radiación electromagnética con una energía tal que no sólo pueden provocar cambios en las moléculas, sino también en la estructura de los átomos que conforman esa molécula, produciendo ionización del átomo, por lo que se denominan radiaciones ionizantes (5).

Las unidades de medición están en relación a la dosis absorbida y se mide en Gray (Gy). El impacto biológico en el tejido, o dosis equivalente, se mide en Sieverts (Sv) y varía dependiendo del tipo de radiación. Con Rayos $X$ el factor de corrección es cercano a 1.0, por lo que la dosis equivalente se considera igual a la dosis absorbida. La unidad final de absorción de radiación es la dosis efectiva (mSv en imágenes diagnósticas), ésta corresponde a la suma de las dosis de los órganos multiplicados por un factor de corrección relacionado con la radiosensibilidad del órgano expuesto (2).

Las TC actuales entregan al final del examen un informe de dosis con el CTDI vol ( Computed Tomography Dose Index volume) y DLP (Dose Lenght Products).

El CTDI vol es un parámetro estandarizado para medir la radiación liberada por la TC, su unidad de medida es Gy. Pero éste no determina la dosis recibida por el paciente. Da información de la radiación usada en el estudio y es útil para comparar protocolos de distintas instituciones, y también 
comparar estudios realizados con diferentes TC $(2,3)$.

El DLP es el producto de CTDIvol (mGy) y la longitud del barrido (cm). Su unidad es $\mathrm{mGy}^{\star} \mathrm{cm}(2)$.

Como la dosis recibida por el paciente no sólo depende de la radiación emitida por la TC sino también del tamaño del paciente, se desarrolló un nuevo factor de conversión en base al CTDIvol, el SSDE (size-specific dose estimates)(3).

\section{RADIACION DE BASE VS RADIACIÓN DIAGNÓSTICA}

La radiación que recibimos proviene de dos grandes fuentes, la natural y la producida por el hombre. La principal fuente de radiación natural son la cósmica y de la biosfera terrestre. La radiación producida por el hombre incluye la ocupacional, industrial y médica (Figura1).

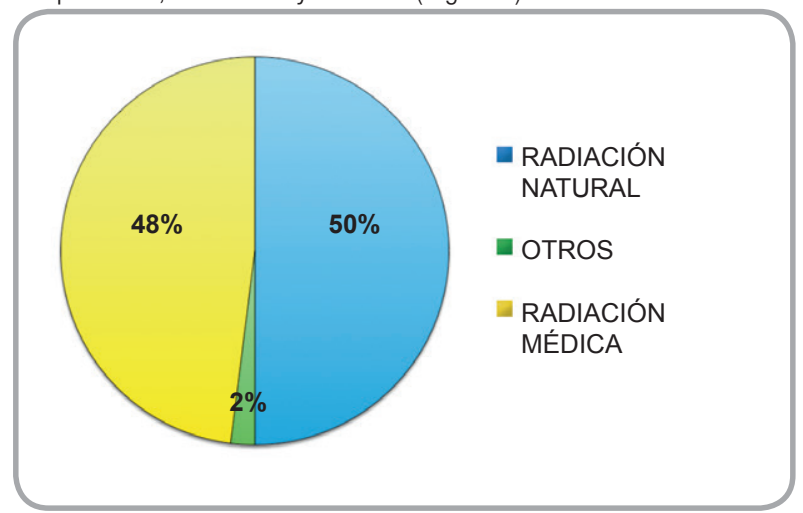

Figura 1. Porcentaje de dosis efectiva colectiva.

Fuente: National Council on Radiation Protection Measurements (6).

Si desglosamos la radiación médica en los distintos usos, nos encontramos que el mayor porcentaje de radiación proviene de la Tomografía Computada (Figura 2)

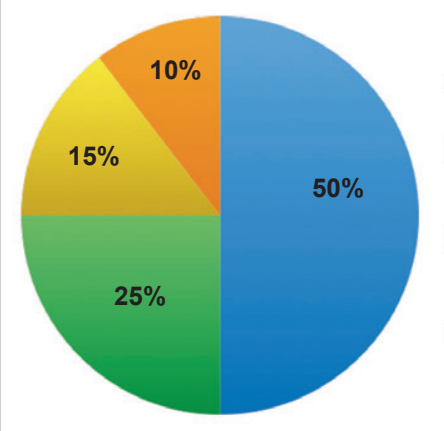

TAC

- MEDICINA NUCLEAR (MN)

- FLUOROSCOPIA INTERVENCIONAL (FI)

- RADIOLOGÍA CONVENSIONAL (RC)

Figura 2. Porcentajes de dosis efectiva colectiva proveniente de

Radiación de uso médico.

Fuente: National Council on Radiation protection and measurements 2006 (6).
El último reporte que midió la exposición a radiación ionizantes en la población de Estados Unidos advirtió un aumento de 7 veces de la radiación base desde los inicios de 1980 a 2006 (7), siendo el factor más importante el aumento de la exposición a radiación médica cerca a un 600\%.

El promedio de dosis efectiva de radiación de base es de $3 \mathrm{mSv}$ al año (varía según el lugar donde vivamos). Comparándola con distintos exámenes se hace más evidente la importancia del CT en la radiación médica (Tabla 1).

\begin{tabular}{|l|c|c|}
\hline RX TÓRAX & Dosis Efectiva (mSv) & Años de Radiación Natural \\
\hline TC TÓRAX & 0.1 & 10 días \\
\hline TC CEREBRO & 2 & 2 años \\
\hline PET/CT & 25 & 8 meses \\
\hline
\end{tabular}

Tabla 1: Promedio dosis efectiva según método. Dosis Efectiva (mSv)

\section{BIOLOGIA DE LA RADIACIÓN}

Los efectos biológicos de la radiación son debidos principalmente al daño del ADN. Los rayos $X$ son absorbidos por el individuoy le dan la energía del fotón a un electrón. El electrón golpea directamente en el ADN produciendo un quiebre de la hebra (acción directa). Cuando el electrón golpea una molécula de agua, H2O produce un radical hidroxilo el cual a su vez produce una ruptura en las hebras de ADN (acción indirecta) (5).

La doble hélice de ADN se mantiene por uniones de hidrógeno. Con dosis menores de radiación se producen daños en sólo una hebra del ADN, reparándose con la hebra contraria. Si la rotura se produce en ambas hebras el resultado es la muerte celular o nuevas uniones cromosómicas. Dos importantes cambios cromosómicos no letales son las translocaciones simétricas, con el potencial de activar oncogenes (como ocurre en linfoma de Burkitt y algunos tipos de leucemia) y pequeñas deleciones (5).

Los efectos de las radiaciones se clasifican en estocásticos y determinísticos. El efecto estocástico no depende de la dosis, pero al incrementar la dosis aumenta la probabilidad de que ocurran. Cuando la célula dañada es una célula somática el efecto se manifiesta en el individuo que recibió la radiación (efectos carcinogénicos). Si la célula dañada es una célula germinal se manifiesta en la herencia (efectos heredables). Por otro lado, están los efectos determinísticos que aumentan su severidad a mayor dosis y se expresan con dosis altas de radiación, por ejemplo el desarrollo de cataratas (5). 


\section{EFECTOS HEREDABLES}

Han habido muchos estudios buscando los posibles efectos heredables secundarios a radiación. El United Nations Scientific Committee on the Effects of Atomic Radiation (UNSCEAR 2013) (8) concluyó que no se habían identificado efectos heredables en humanos debido a la exposición a radiación, en especial en la descendencia de sobrevivientes de la bomba atómica. En la última década estudios de niños y adolescentes sobrevivientes de cáncer gonadal con dosis alta por radioterapia, no se encontró evidencia de aumento de mutaciones, inestabilidad cromosomal, anomalías congénitas ni aumento de riesgo de cáncer en descendientes de padres expuestos a radiación (5).

\section{EFECTOS BIOLÓGICOS DE LA RADIACION EN NIÑOS VS ADULTOS}

La diferencia de los efectos biológicos de la radiación ionizante entre adultos y niños recae en tres factores que son: la radiosensibilidad, la expectativa de vida y la exposición a la radiación.

Durante la infancia los órganos y tejidos se distribuyen en forma diferente a los adultos. La médula ósea, que en los adultos está compuesta principalmente de grasa, en los niños tiene gran proporción de médula roja. Los tejidos están en proceso de proliferación celular con mayor número de mitosis, lo que los hace más susceptibles. Los tejidos más radiosensibles durante la niñez son tiroídeo, pulmonar, mamario y médula ósea.

Así, los tumores malignos se desarrollan muchos años después de la exposición, por lo que los adultos pueden fallecer por otras causas antes que el cáncer inducido por radiación se exprese, hecho que hace la diferencia en los niños por tener una mayor expectativa de vida.

En el caso de la TC la dosis recibida por un niño, aún con parámetros modificados es proporcionalmente mayor que la de un adulto. Esto se debe al menor espesor de los órganos, hay una mayor proporción de órganos en relación a la masa total del individuo y los órganos están más cercanos entre ellos (7, 8-12).

\section{TC EN PEDIATRIA}

En USA el $10 \%$ de los exámenes radiológicos son TC y su uso se ha incrementado en un 10\% por año. A pesar de estas cifras, es el examen que más contribuye a la dosis colectiva de radiación, Ilegando a ser entre el 50 al $67 \%$ en centros de referencia en USA (6)

Con el desarrollo de los tomógrafos de última generación ( multicorte), los exámenes se han hecho cada vez más rápidos, disminuyendo la necesidad de anestesia y sedación en los niños. También con la mayor resolución han aumentado las indicaciones, y junto a esto, aún mayor dosis de radiación.

\section{EVIDENCIAS DEL EFECTO CARCINOGÉNICO}

La primera gran evidencia de cáncer inducido por radiación fue el estudio de los sobrevivientes de la bomba atómica. El estudio fue un seguimiento por más de 60 años, involucrando a más de 100.000 personas. Lo más Ilamativo fue el exceso de cáncer en pacientes expuestos a dosis baja de radiación (5mSv a 200 mSv). Además quedó claramente demostrado que los más afectados eran los más jóvenes, siendo un recién nacido 10 a 15 veces más sensible a la radiación que un adulto. También se demostró que las mujeres tenían un riesgo mayor que los hombres principalmente por el aumento de cáncer de mamas.

Un estudio cohorte retrospectivo de 180.000 pacientes menores de 22 años de Inglaterra y Escocia examinados con TC , mostró una relación positiva entre la dosis del TC y el exceso de riesgo relativo de leucemia y tumores cerebrales, alcanzando casi el triple de riesgo de leucemia con dosis acumulada de $50 \mathrm{mGy}$ y el triple de riesgo de tumores cerebrales con dosis acumulada de 60mGy. Por la baja incidencia de estos cánceres se estima como un caso extra de cada uno (leucemia y tumor cerebral ) por cada 10.000 TC (11).

Otra publicación reciente de Mathews et al, en una población de casi 11 millones de niños y jóvenes de Australia, de los cuales 680.000 habían sido sometidos a uno 0 más scanner, reveló un aumento de la incidencia de cáncer. Se analizó la proporción de tasa de incidencia de cáncer (IRR, incidence rate ratios) de expuesto y no expuestos separándolos por edad, sexo y año de nacimiento (7).

La incidencia de cáncer total fue $24 \%$ mayor en relación a los no expuestos, se observó también una relación directa con la dosis, aumentando el IRR de $0.16(0.13-1.19)$ por cada TC adicional y fue aún mayor después de la exposición de los más jóvenes.

Entre los cánceres sólidos que más aumentaron están los de órganos digestivos, melanoma, tejidos blandos, genital femenino, tracto urinario, cerebro y tiroides. y, de los no sólidos, las leucemias, mielodisplasias y linfoproliferativos.

La tasa de incidencia absoluta en exceso de todos los cánceres combinados fue de 9,38 por 100.000 personas a Diciembre 2007.

Estos estudios cambiaron la percepción de que el riesgo de la TC era prácticamente inexistente, lo que nos debe hacer reflexionar con mayor seriedad en las medidas que debemos tomar para reducir al máximo este riesgo (7,10-12).

\section{FORMAS DE MINIMIZAR LA RADIACION PROVENIENTE DE TAC EN PEDIATRIA $(3,10)$}

La responsabilidad de disminuir la radiación expuesta en niños recae en varios actores desde las empresas fabricantes de los tomógrafos, organizaciones gubernamentales, sociedades científicas, tecnólogos y radiólogos, pediatras, médicos que solicitan los exámenes y también los padres.

Podemos resumir las medidas en:

1) Indicación del Examen:

- Rigurosa justificación del examen, priorizando aquellos que no aportan radiación ionizante (ecografía o RM)

- Aportar la mayor cantidad de antecedentes previo al exámen, manteniendo una adecuada comunicación entre el clínico y el radiólogo. Evitar repetir exámenes por duplicidad de 
órdenes o por pérdida, educando también a los padres para que tengan un rol activo en el registro y cuidado de los exámenes que se realizan sus hijos.

2) Protocolos adecuados para niños.

1) Limitar a la menor área posible la región estudiada.

2) Ajustar la exposición según el órgano, utilizando menor mA o kV en estudios esqueléticos 0 pulmón y también en seguimientos.

3) Adecuar los protocolos en base a tamaño y peso del paciente.

\section{3) Calidad de imagen}

Evitar exámenes de alta resolución (mayor radiación), utilizando sólo la necesaria para realizar diagnóstico.

4) Notificación de dosis, alertas para tomógrafos y almacenaje de datos.

En Estados Unidos los nuevos equipos deben cumplir con standard de dosis exigido y deberán indicar la dosis de radiación del paciente (DRL $=$ Diagnostic Reference Level) que es el primer paso en la optimización de procesos.

Los actuales TC se pueden configurar para detectar parámetros no adecuados de CTDIvol o DLP antes que se realice el estudio, dando una alerta para no sobrepasar los valores preasignados. (Dose Check Standard).

El registro y almacenaje de informes de dosis que aportan los CT actuales nos permitirá comparar nuestros protocolos con los estándares internacionales, logrando óptimos niveles de radiación.

\section{CONCLUSIÓN}

No hay duda que los métodos de imágenes son una herramienta importantísima en la práctica clínica. Con los últimos estudios de los efectos de radiación de la TC se descartan las teorías que proponían que por ser incidencias tan bajas no eran detectables y probablemente inexistentes. Ya sabiendo que el riesgo sí existe, aunque bajo, debe mantenernos en permanente alerta para buscar formas de reducción de la dosis de radiación a que se exponen los niños, sin olvidarnos que es un excelente método de imagen y que si clínicamente se justifica los beneficios serán mucho más amplios que los costos.

\section{El autor no presenta conflicto de intereses.}

\section{REFERENCIAS}

1. Scatliff JH, Morris PJ. From Roentgen to magnetic resonance imaging: the history of medical imaging. N C Med J. 2014 ;75:111-3

2. American Association of Phisicists in Medicine. Report of AAPM Task Group 204. Size-Specific Dose Estimates (SSDE) in Pediatric and Adult Body CT Examinations. ISBN 978-1-936366-
08-8, ISSN 0271-7344, 2011, p 1-23

3. Zacharias C, Alessio AM, Otto RK, Lyer RS, Philips GS, Swanson J0, Thapa MM. Pediatric CT strategies lower radiation dose. AJR 2013; 200:950-956

4. Xiaochuan Pan X, Siewerdsen J, La Riviere PJ, Kalender WA. Anniversary Paper: Development of X-ray computed tomography: The role of Medical Physics and AAPM from the 1970s to present. Med Phys 2008;35:3728-379

5. Hall E. Radiation Biology for Pediatric Radiologists. Pediatr Radiol 2009;39 Suppl 1: S57- S64

6. Chen J, Moir D. An estimation of the annual effective dose to the Canadian population from medical CT examinations. J Radiol Prot. 2010 30:131-7

7. Mathews J, Forsythe A, Brady Z, Butler M, Goergen S, Byrnes $G$, Giles $G$ et al. Cancer risk in 680,000 people exposed to computed tomography scans in childhood or adolescence : data linkage study of 11 millon Australians. BMJ 2013;346:f2360 doi: 10.1136/bmj.f2360

8. Hall E. Radiation Biology for Pediatric Radiologists. Pediatr Radiol 2009;39 Suppl 1: S57- S64

9. United Nations Scientific Committee on the Effects of Atomic Radiation (UNSCEAR 2013) Annex B

10. International Atomic Energy Agency (IAEA) 2012. Radiation Protection in Paediatric Radiology. Safety Reports N071 Appendix 11. Brody AS, Frush DP, Huda W, Brent RL. Radiation risk to children from computed tomography. Pediatrics 2007;120:677682

12. Pearce M, Salotti J, Litlle M, McHugh K, Lee C, Pyo Kim $\mathrm{K}$ et al. Radiation exposure from CT scans in childhood and subsequent risk of leukaemia and brain tumours: a retrospective cohort study. Lancet 2012; 380:499-505 